\title{
"Is There An App For That?" Orthopaedic Patient Preferences For A Smartphone Application
}

Jonathan R. Dattilo1; Daniel J. Gittings'; Matthew Sloan; William M. Hardaker'; Matthew J. Deasey²; Neil P. Sheth ${ }^{1}$

${ }^{1}$ University of Pennsylvania, Orthopaedic Surgery, Philadelphia, Pennsylvania, United States;

${ }^{2}$ Lewis Katz School of Medicine at Temple University, Philadelphia, Pennsylvania, United States

\section{Keywords}

Smartphone, telemedicine, e-health, orthopaedic surgery, internet

\section{Summary}

Background: Patients are seeking out medical information on the Internet and utilizing smartphone health applications ("apps"). Smartphone use has exponentially increased among orthopaedic surgeons and patients. Despite this increase, patients are rarely directed to specific apps by physicians. No study exists querying patient preferences for a patient-centered, orthopaedic smartphone application.

Objectives: The purpose of this study is to 1) determine Internet use patterns amongst orthopaedic patients; 2) ascertain access to and use of smartphones; and 3) elucidate what features orthopaedic patients find most important in a smartphone application.

Methods: We surveyed patients in an orthopaedic practice in an urban academic center to assess demographics, access to and patterns of Internet and Smartphone use, and preferences for features in a smartphone app.

Results: A total of 310 surveys were completed. Eighty percent of patients reported Internet access, and $62 \%$ used the Internet for health information. Seventy-seven percent owned smartphones, $45 \%$ used them for health information, and $28 \%$ owned health apps. Only $11 \%$ were referred to an app by a physician. The highest ranked features were appointment reminders, ability to view test results, communication with physicians, and discharge instructions. General orthopaedic information and pictures or videos explaining surgery were the 2 lowest ranked features. Seventy-one percent of patients felt an app with some of the described features would improve their healthcare experiences, and $40 \%$ would pay for the app.

Conclusions: The smartphone is an under-utilized tool to enhance patient-physician communication, increase satisfaction, and improve quality of care. Patients were enthusiastic about app features that are often included in patient health portals, but ranked orthopaedic educational features lowest. Further study is required to elucidate how best to use orthopaedic apps as physician-directed educational opportunities to promote patient satisfaction and quality of care.

\section{Correspondence to:}

Neil P. Sheth, MD

University of Pennsylvania

800 Spruce Street, $1^{\text {st }}$ Floor Cathcart Building

Philadelphia, PA 19107, USA

Phone: 215-662-3340

Fax: 215-349-5890

Email: neil.sheth@uphs.upenn.edu

\section{Appl Clin Inform 2017; 8: 832-844}

https://doi.org/10.4338/ACI-2017-04-RA-0058

received: 9. April 2017

accepted in revised form: 27. June 2017

published: August 16, 2017

Citation: Dattilo JR, Gittings DJ, Sloan M, Hardaker

WM, Deasey MJ, Sheth NP. "Is There An App For That?"

Orthopaedic Patient Preferences For A Smartphone Ap-

plication. Appl Clin Inform 2017; 8: 832-844

https://doi.org/10.4338/ACI-2017-04-RA-0058 


\section{Background and Significance}

Patient satisfaction with their healthcare experience is becoming an integral part of quality of care measures and reimbursement. Patient satisfaction has the potential to influence surgeon income, employment, and incentives [1,2]. In a survey of over 700 medical professionals, $27 \%$ of providers reported their income was partly dependent on patient satisfaction scores, and $16 \%$ admitted that their employer had threatened their employment if poor satisfaction scores were earned [3]. The most popular patient satisfaction surveys are produced by Press Ganey (Press Ganey Associates Incorporated, South Bend, IN) and are distributed in nearly $50 \%$ of all hospitals and over 10,000 health care organizations in the United States [2].

Traditionally patients have sought the opinions of medical professionals to obtain common health information, engaging in a now historic, paternalistic model of medicine. The modern era of healthcare has embraced what has been termed the "e-patient" in a 2009 study, demonstrating that $61 \%$ of patients employ the Internet as a source of health information. This represents a burgeoning niche for software developers, who have seized the opportunity to appeal to patients seeking out medical knowledge and more active involvement in their healthcare, by creating Internet websites and smartphone applications. In 2007 Apple (Apple Incorporated, Cupertino, CA) released the iPhone and its novel iTunes store, which enabled developers to create and distribute apps to consumers worldwide. Google (Google Incorporated, Mountain View, CA) soon followed suit, and as of 2015 , there were approximately 100,000 health-related apps on the two major smartphone operating systems, Apple's iOS and Google's Android [4].

Amongst orthopaedic surgeons, smartphone apps have been embraced in clinical practice. Andrawis et al. conducted a study from 2010 to 2014, demonstrating an increase in use of smartphone apps in the clinical setting from $60 \%$ to $84 \%$ by orthopaedic trainees and $41 \%$ to $61 \%$ for orthopaedic attendings. Furthermore, $70 \%$ of orthopaedic surgeons believed that their institution should support mobile device use [5].

Orthopaedic surgery is the specialty with the greatest number of publications on the topic of mobile apps and app validation [5-8]. Numerous orthopaedic apps have been developed for physician use in the clinical environment, including arthroplasty $[9,10]$, pediatrics [11], sports [12-14], and spinal surgery $[15,16]$.

Health app utilization is also highly prevalent amongst patients. VonHoltz et al. demonstrated that $71 \%$ of patients owned smartphones, $44 \%$ of whom used health apps [17]. However, only $2 \%$ of these apps were recommended to patients by a healthcare provider, suggesting that the current state of app development is limited to dissemination of health information rather than as a means of facilitating patient-provider communication.

The limitations in developing physician-directed patient education apps may be in part due to a paucity of objective data assessing what features patients truly desire in a health app. A study of rheumatoid arthritis patients revealed several disease-specific needs in a mobile application that may not be apparent to providers or developers [18]. Cho et al. attempted to create a patient-friendly educational app for cardiology, but did not directly solicit patient preferences [19]. Perhaps the most enlightening examination comes from an outpatient pharmacy, which found that there were 3 themes most important for a patient's mobile application experience: facilitation of a convenient pharmacy encounter; features that support the self-management of the patient's health; and personalized, timely access to the pharmacist [20].

Several disadvantages do exist regarding the use of smartphones and mobile health applications. Lack of oversight in application development has caused concern regarding the quality of content and security of information transmitted in mobile health apps. While medical apps can empower patients through self-education, false information or misleading opinions can also be detrimental and may present an obstacle the physician must overcome to establish rapport with the patient [21]. In the United States, the Food and Drug Administration has suggested the need for regulation of app content, though no peer review process currently exists [22]. Similarly, concerns have been raised over patient privacy and the security of data transmission through mobile apps. A study of Emergency Department physicians demonstrated the utility of the WhatsApp application to transmit information to consulting physicians, particularly orthopaedics [23]. However, there is no barrier to the transmission of identifiable patient information, and others have demonstrated poor 
compliance with electronic transmission of confidential patient data, particularly amongst surgical trainees [24]. These concerns highlight the importance of research aimed at directing application development by providing guidance to what is most important to patients and physicians. Application development should foster a partnership between physicians and software developers to ensure the quality of content and security of apps to safely promote physician-directed patient education.

\section{Objectives}

To date, no study exists that addresses the question of patient preferences for an orthopaedic smartphone application. The purpose of the current study is to evaluate patients in an elective orthopaedic sub-specialty practice to

- determine Internet use patterns among patients;

- ascertain access to and use of smartphones; and

- elucidate what features patients find most important in an orthopaedic smartphone application.

\section{Methods}

Following institutional review board approval, a 10-question survey was created and administered to all patients presenting to an adult orthopaedic arthroplasty practice in an urban academic center. A pilot survey was distributed for 2 weeks, during which time patients were asked to provide feedback in real time and returned surveys were examined to ensure patient comprehension and correct survey completion. Based on feedback from the pilot survey, a final survey was created (Appendix 1). The results of the pilot study period were not counted or included in the analysis. Final surveys were administered over a 3-month period. Inclusion criteria included any adult patient, whether established or new, presenting to the clinic. Patients under the age of 18 were excluded as the practice treats only adult patients. There were no other exclusion criteria. Patients received a survey upon check-in to the clinic and could return completed surveys to the clinic staff or anonymously at the front desk at the conclusion of their visit. All surveys were anonymous and optional, and no monetary incentive was provided for survey completion.

The first portion of the survey assessed basic demographic information, including age, gender, race, socioeconomic status, and education level. The second portion of the survey elicited whether the patient had home Internet access and/or owned a smartphone, and if so whether they had ever used or been referred to these mediums for health-related information. Patients were then asked to rate 10 theoretical features that could be incorporated in an orthopaedic smartphone app using a 5-point Likert scale ( Table 1).

\subsection{Statistical Analysis}

Surveys were collected and results were recorded in a Microsoft Excel spreadsheet (Microsoft Corporation, Redmond, WA). Statistical analysis was performed using StataIC Version 14.0 (StataCorp, College Station, TX). All data was subject to descriptive statistical analysis yielding frequency scores for categorical data. Statistically significant differences between variables were then determined by conducting inferential statistical analysis using the Chi-Square test. A p-value less than or equal to 0.05 was considered statistically significant, as this would indicate differences between groups had less than $5 \%$ likelihood of being due to chance alone.

Patient preferences were assessed using a rank order calculation. Each proposed feature was ranked 1 through 5 using a traditional Likert scale. Each rank was multiplied by the amount of times patients chose that rank. A score for each feature was calculated based on the sum of each individual rank for that feature. The final list of patient preferences was based on each features' summed scores. 


\section{Results}

Three hundred seventy-two surveys were administered from July to September 2016. Nineteen patients preferred not to respond and forty-three did not return their survey, resulting in 310 completed surveys and a response rate of $83 \%$. Demographic data is demonstrated in Table 2. Sixtyone percent of response rate of $83 \%$. Demographic data is demonstrated in Table II. Sixty-one percent of respondents were female while $39 \%$ were male. The most common age range was 56-70 years (43\%), followed by 41-55 (31\%). Most patients were Caucasian (49\%) or African American (40\%). The most common income reported was less than $\$ 30,000(33 \%)$, followed by greater than $\$ 75,000$ (21\%). Fifty-four percent of patients had completed high school, obtained their general educational development certificate (GED), or completed some but not all of college. Thirty-five percent had completed college or obtained a graduate degree.

\subsection{Internet Access and Use for Health Information}

Eighty percent of patients reported access to the Internet at home ( Table 3). Neither gender nor age was associated with access to the Internet at home. Race was significantly $(p=0.023)$ associated with Internet access, with a higher proportion of Caucasians having access than African Americans. Higher income $(\mathrm{p}<0.001)$ and increasing level of education $(\mathrm{p}<0.001)$ were also significantly associated with home Internet access ( $\triangleright$ Table 4 ). Sixty-two percent of patients had used the Internet to obtain health-related information. Amongst patients who reported using the Internet to obtain health information, there were no differences between males and females. However, younger age $(\mathrm{p}=0.045)$, higher income $(\mathrm{p}<0.001)$, and increasing education $(\mathrm{p}<0.001)$ were significantly associated with seeking online health information. A higher proportion of Caucasians than African Americans also sought medical information online $(\mathrm{p}<0.001)$ ( Table 4$)$.

\subsection{Smartphone Access and Use for Health Information}

Seventy-seven percent of respondents owned a smartphone. Amongst smartphone owners, Android (55\%) was the most popular, followed by the iPhone (44.5\%). Microsoft Windows phone made up a very small $(0.5 \%)$ percentage, and no patient owned a Blackberry ( Table 3$)$. Forty-five percent of smartphone users had accessed health-related information from their phone, and $28 \%$ had downloaded health-related apps. However, only $11 \%$ of patients had ever been referred to an app by a physician. Younger age $(\mathrm{p}<0.001)$, higher income $(\mathrm{p}<0.001)$, and increasing education $(\mathrm{p}=0.008)$ were associated with smartphone ownership. Gender and race were not significantly predictive of owning a smartphone. Amongst smartphone owners, younger age $(\mathrm{p}<0.001)$, higher income $(\mathrm{p}=0.01)$, and increasing education $(\mathrm{p}<0.001)$ were significantly associated with obtaining medical information via smartphone. Neither race nor gender was associated with using smartphones to access health information ( Table 5).

\subsection{Patient Preferences for App Features}

Patient preferences for smartphone application features are summarized in $>$ Table 6 . The four highest ranked features were appointment reminders, ability to view results of tests or procedures, ability to communicate with physicians or treatment team, and easy access to discharge instructions. The two lowest ranked features were information about general orthopaedic conditions and animated content, including pictures or videos, about orthopaedic procedures. Overall, $71 \%$ of patients felt that an orthopaedic smartphone application would improve their healthcare experiences, and $40 \%$ were willing to pay for an app that incorporated some or all of their preferences. The interquartile range for patients willing to pay for the app was between $\$ 1$ and $\$ 5$. 


\section{Discussion}

Patient satisfaction scores are increasingly used to determine quality of care, and have the potential to influence reimbursement from the Center for Medicare and Medicaid Services [2]. However, quality as measured by physicians or traditional orthopaedic outcome measures are not the same as those included in typical patient satisfaction questionnaires. For instance, the amount of communication with patients' providers was shown to be a statistically significant contributor to Press Ganey satisfaction scores in an orthopaedic surgery spine clinic [25], but would not typically be assessed with traditional patient-reported spine outcomes surveys such as the Cervical Spine Outcomes Questionnaire or Myelopathy Disability Index [26]. Neglecting patient satisfaction elements detracts from the quality of care delivered, and moreover may risk the loss of substantial reimbursement. It is therefore paramount that orthopaedic surgeons actively seek to understand factors that may affect their patients' satisfaction with their healthcare experiences.

The smartphone represents a powerful tool that can be utilized to enhance patient satisfaction. However, it is a currently under-utilized means of appealing to patient satisfaction and enabling patients to take active roles as participants in their healthcare. Both orthopaedic surgeons and patients are readily embracing health apps, but the utilization of apps as physician-directed patient educational opportunities has not yet been achieved. This is likely due to the fact that current apps are either designed for physicians or for patients, as no study has answered the question of what patients want in an orthopaedic smartphone application. Therefore, the purpose of the current study was to

- determine Internet use patterns among patients;

- ascertain access to and use of smartphones; and

- elucidate what features patients find most important in an orthopaedic smartphone application.

Internet use to access health information has become common in the modern era of the "e-patient." In our study, $80 \%$ of patients reported access to the Internet at home. The results of today's orthopaedic patients are slightly higher, as expected due to increased Internet usage, compared to a 2009 study in which $70 \%$ of households had Internet access [27]. Sixty-two percent of our patients had used the Internet to obtain health-related information, which is also consistent with prior reports $[28,29]$. It has been estimated that up to $33 \%$ of American adults report musculoskeletal complaints at any given time[30], which may in part explain the propensity to access orthopaedic information. Female gender, younger age, increased socioeconomic status, and higher education have previously been associated with increased health-related Internet use [28, 31]. In contrast to prior reports, our study did not find significant associations between gender and Internet access, nor the likelihood of accessing health information via the Internet. Race was significantly associated with Internet access and Internet use for health information. Consistent with prior studies, our analysis indicates that increasing socioeconomic status and education level were significantly associated with both access to the Internet and its use to acquire medical information.

Smartphone use was very common in our arthroplasty population. Seventy-seven percent of patients surveyed reported owning a smartphone. Fifty-five percent of patients owned Android smartphones, while $45 \%$ owned an iPhone. This is consistent with 2015 market estimates demonstrating 59.1\% market share for Android and 39.1\% for Apple, with Blackberry and Windows comprising extremely small fractions of the smartphone domain [32]. Forty-five percent of existing smartphone users reported having accessed health information from their phones, and 28\% regularly used health apps. While the proportion of patients utilizing health apps in our arthroplasty population is lower than previously reported in younger populations [17], our respondents indicated a high degree of interest in app utilization. Seventy-one percent of patients felt that an app would improve their healthcare experience, and $40 \%$ would pay for this app. The interquartile range that patients would spend was between $\$ 1$ and $\$ 5$, suggesting a viable price point for future patient education apps. However, only $11 \%$ of patients had ever been referred to an app by a physician. These results indicate that while many patients are seeking health information in the form of Internet content or dedicated apps, and are even willing to pay out-of-pocket for this information, there is not yet a bridge between the patient and provider to recommend apps as a means for communication or education.

Analysis of patient preferences for features in an orthopaedic app revealed the importance of personalized healthcare information rather than mere access to medical information. The highest 
ranked features were appointment reminders, the ability to view results of tests or procedures, communication with the provider or treatment team, and easy access to discharge instructions. Together, these features more closely resemble a patient access or health portal, which is a feature offered through the electronic medical record at some institutions to facilitate patient access to health records and communication with providers [33]. These results, particularly the emphasis on access to discharge instructions, suggest that the communication needs of arthroplasty patients may differ from the needs of patients with chronic medical problems. A patient with joint arthritis is typically managed first with non-operative interventions such as physical therapy and medication, and if or when these modalities become insufficient the physician and patient may discuss surgery. During this spectrum of treatments, it is possible that the communication needs of the patient may change. The patient may have a greater desire to communicate with their provider if they are considering surgery or in the immediate post-operative recovery period. Further research is warranted to elucidate any temporal effects to better design apps or improve health portals to meet the needs of orthopaedic patients.

Information about general orthopaedic conditions and educational animated content explaining orthopaedic procedures were the two features ranked lowest by patients. This is particularly insightful, as these features commonly form the hallmark of content in patient education apps. This discrepancy is likely in part due to the fact that no one has previously asked patients what features they would prefer in an app. Instead, physicians and developers have presumed to know what content patients will find most helpful. While the "e-patient" may want a more active role in their healthcare, it appears that the most important features involve facilitating communication with their provider and ease of follow-up appointments and instructions. It is possible that the currently available orthopaedic patient education content, when viewed solely by patients without guidance or personalized explanation by their physicians, may raise more questions than it answers. The needs of patients may also change depending on the type of treatment they receive. For instance, a patient managed with physical therapy may not have the desire for information from an app because they have personal guidance from their therapist multiple times per week. However, a patient recovering from surgery may have greater desire for information about the normal post-operative recovery milestones which could form the foundation for a mobile app. It is also possible that because physicians are not actively referring patients to specific healthcare apps, patients may be reticent to trust online information or otherwise be simply overwhelmed by the numerous patient education apps currently available. These results underscore the importance of the personal dialogue between a patient and physician.

This study has several limitations. First, this is a single institution and single surgeon study and therefore susceptible to flaws and biases in data capture and collection. However, data was prospectively collected and all patients were given an opportunity to complete the survey, resulting in a response rate of $83 \%$. This minimizes the risk of sampling bias. Second, this study was conducted in an urban, academic practice and thus generalizability to alternate practice settings may be limited. However, there were no racial disparities observed between smartphone and non-smartphone user groups, and differences in socioeconomic status and education level are consistent with prior reports $[28,31]$, suggesting the risk of potential bias is low. Additionally, joint arthroplasty is generally an elective surgery in which patients are able to choose their surgeon and timing of surgery. However, it is unclear if these same patterns or preferences would apply to emergent or non-elective settings. Finally, surveys were distributed with defined rather than open-ended questions to query patient preferences. As the surveys were distributed during the clinic visit, we also limited the length to 2 pages to avoid delays in clinical care. The results from the survey are necessarily more limited than an open-ended means such as a focus group, which would enable further clarification and follow up questions. However, we attempted to refine the survey structure and its content through an initial pilot period with patient input to maximize our data collection, which could now be used to secure funding for focus groups or patient interviews. 


\section{Conclusion}

This study presents an evaluation of the Internet and smartphone usage patterns of patients in an adult reconstructive orthopaedic surgery practice, and queries patient preferences for features in a smartphone app. Responses indicate that patients are eager to embrace smartphone apps as a means of taking active roles in their healthcare, and that smartphones are an under-utilized means to promote these roles. Patients indicated that they value features that facilitate communication with their providers and ease of access to their personal health information more than general orthopaedic information or pictures or videos explaining surgery. While these results indicate that patients may be eager to embrace patient portal institutional apps, further study is required to elucidate why patients were less enthusiastic about orthopaedic specific patient education apps. Future studies may benefit from more open-ended patient queries, such as focus groups, to more fully understand any reservations surrounding orthopaedic educational content. This information will be useful to both enlighten the practicing orthopaedist as well as software developers in order to successfully create education apps for patients that will have widespread appeal and may be incorporated into the dialogue of patient-physician communication to promote patient satisfaction and quality of care.

\section{Multiple Choice Questions}

Which of the following are potential implications of patient satisfaction scores in the United States?

A) Influence provider income

B) Influence provider employment

C) Provide feedback to providers on quality of care delivered

D) All of the above

Answer: D. As discussed in the introduction and discussion, patient satisfaction surveys are becoming more influential in rating the quality of care delivered. Several studies have identified these surveys as having the potential to influence provider income, either negatively or positively, and even affect employment status if poor results are earned.

Which of the following are referenced in the article as concerns about mobile health applications?

A) Cost and privacy of patient information

B) Quality of app content and privacy of patient information

C) Privacy of patient information and availability across multiple operating systems

D) Cost and availability across multiple operating systems

Answer: B. At the present time there is no peer review process to ensure quality of health content in mobile applications. Similarly, there is no universal software standard to inhibit the transmission of patient protected information, and rather the onus is on the provider to adhere to privacy laws. Both of these issues have been cited as concerns about application use in healthcare.

\section{Clinical Relevance Statement}

Smartphone use, including healthcare applications, has exponentially increased among both orthopaedic surgeons and patients. Rarely, however, are patients referred to apps by their physicians or use them as an integral part of their healthcare experience. Our study demonstrates that patients are eager to embrace smartphone applications as a means of taking active roles in their healthcare, and that smartphones are an under-utilized means to promote these roles.

\section{Conflict of Interest}

The authors report no conflicts of interest related to this work.

\section{Human Subjects Protections}

This study was conducted in accordance with the regulations of the US Health Insurance Portability and Accountability Act (HIPAA) and in conjunction with Institutional Review Board (IRB) approval. 
Table 1 Features in an orthopaedic smartphone app

\begin{tabular}{|l|l|}
\hline Feature & Likert Scale \\
\hline Finding a doctor: information about hospital or physicians & 12345 \\
\hline Making or changing appointments & 12345 \\
\hline Reminders before next appointment or surgery & 12345 \\
\hline Review results of tests, procedures, labs, or xrays/CT/MRI & 12345 \\
\hline Communication with physician team (texts, emails) & 12345 \\
\hline Medication information & 12345 \\
\hline Information about general orthopaedic problems & 12345 \\
\hline Pictures/videos explaining surgery & 12345 \\
\hline Physical therapy exercise instructions/pictures/videos & 12345 \\
\hline Discharge or after-surgery instructions & 12345 \\
\hline
\end{tabular}

Table 2 Respondent Demographics; *USD = United States Dollar

\begin{tabular}{|c|c|c|c|}
\hline & Respondents (\%) & & Respondents (\%) \\
\hline \multicolumn{2}{|l|}{ Sex } & \multicolumn{2}{|c|}{ Income (thousands of USD) } \\
\hline Male & 39 & $<30$ & 33 \\
\hline Female & 61 & $30-49$ & 10 \\
\hline \multicolumn{2}{|l|}{ Age (years) } & $50-74$ & 14 \\
\hline $18-24$ & 2 & $>75$ & 21 \\
\hline $25-40$ & 11 & \multicolumn{2}{|l|}{ Education } \\
\hline $41-55$ & 31 & Prefer not to say & 22 \\
\hline $56-70$ & 43 & Less than high school & 9 \\
\hline $71-85$ & 13 & High school or GED & 32 \\
\hline$>85$ & $<1$ & Some college & 22 \\
\hline \multicolumn{2}{|l|}{ Race } & Completed college & 20 \\
\hline African American & 40 & Graduate degree & 15 \\
\hline Caucasian & 49 & Prefer not to say & 2 \\
\hline Asian & 3 & & \\
\hline Hispanic & 4 & & \\
\hline Other & 4 & & \\
\hline
\end{tabular}


Table 3 Summary of Internet and Smartphone Access and Use

\begin{tabular}{|l|l|l|}
\hline & No (\%) & Yes (\%) \\
\hline Access to Internet at home & 20 & 80 \\
\hline Use Internet for health information & 38 & 62 \\
\hline Own a smartphone & 23 & 77 \\
\hline Use Smartphone for health information & 55 & 45 \\
\hline Referred to health app by a physician & 89 & 11 \\
\hline Smartphone Brand & Percent (\%) & \\
\hline Android & 55 & \\
\hline iPhone & 44.5 & \\
\hline Microsoft Windows & 0.5 & \\
\hline Blackberry & 0 & \\
\hline
\end{tabular}

Table 4 Internet Access and Use for Health Information; * USD = United States Dollar

\begin{tabular}{|c|c|c|c|c|c|c|c|}
\hline \multicolumn{4}{|c|}{ Patients with Home Internet Access } & \multicolumn{4}{|c|}{ Internet Use for Health Information } \\
\hline Demographics & No $(\%)$ & Yes $(\%)$ & P Value & Demographics & No $(\%)$ & Yes (\%) & P Value \\
\hline \multicolumn{3}{|l|}{ Sex } & $p=0.202$ & \multicolumn{3}{|l|}{ Sex } & $p=0.527$ \\
\hline Male & 16 & 84 & & Male & 36 & 64 & \\
\hline Female & 23 & 77 & & Female & 39 & 61 & \\
\hline \multicolumn{3}{|l|}{ Age (years) } & $p=0.077$ & \multicolumn{3}{|l|}{ Age (years) } & $p=0.045$ \\
\hline $18-24$ & 17 & 83 & & $18-24$ & 17 & 83 & \\
\hline $25-40$ & 12 & 88 & & $25-40$ & 18 & 82 & \\
\hline $41-55$ & 14 & 86 & & $41-55$ & 35 & 65 & \\
\hline $56-70$ & 22 & 78 & & $56-70$ & 45 & 55 & \\
\hline $71-85$ & 30 & 70 & & $71-85$ & 43 & 57 & \\
\hline$>85$ & 100 & 0 & & $>85$ & 100 & 0 & \\
\hline \multicolumn{3}{|l|}{ Race } & $p=0.023$ & \multicolumn{3}{|l|}{ Race } & $p<0.001$ \\
\hline African American & 28 & 72 & & African American & 51 & 49 & \\
\hline Caucasian & 12 & 88 & & Caucasian & 26 & 74 & \\
\hline Asian & 30 & 70 & & Asian & 86 & 14 & \\
\hline Hispanic & 25 & 75 & & Hispanic & 42 & 58 & \\
\hline Other & 18 & 82 & & Other & 36 & 64 & \\
\hline \multicolumn{3}{|c|}{ Income (thousands of USD) } & $p<0.001$ & \multicolumn{3}{|c|}{ Income (thousands of USD) } & $p<0.001$ \\
\hline$<30$ & 35 & 65 & & $<30$ & 57 & 43 & \\
\hline $30-49$ & 16 & 84 & & $30-49$ & 35 & 65 & \\
\hline $50-74$ & 5 & 95 & & $50-74$ & 24 & 76 & \\
\hline$>75$ & 2 & 98 & & $>75$ & 8 & 92 & \\
\hline \multicolumn{3}{|l|}{ Education } & $p<0.001$ & \multicolumn{3}{|l|}{ Education } & $p<0.001$ \\
\hline Less than high school & 56 & 44 & & Less than high school & 80 & 20 & \\
\hline High school or GED & 31 & 69 & & High school or GED & 54 & 46 & \\
\hline Some college & 7 & 93 & & Some college & 33 & 67 & \\
\hline Completed college & 8 & 92 & & Completed college & 17 & 83 & \\
\hline Graduate degree & 2 & 98 & & Graduate degree & 11 & 89 & \\
\hline
\end{tabular}


Table 5 Smartphone Access and Use for Health Information; *USD = United States Dollar

\begin{tabular}{|c|c|c|c|c|c|c|c|}
\hline \multicolumn{4}{|c|}{ Smartphone Ownership } & \multicolumn{4}{|c|}{ Smartphone Use for Health Information } \\
\hline Demographics & No $(\%)$ & Yes (\%) & P Value & Demographics & No $(\%)$ & Yes $(\%)$ & P Value \\
\hline \multicolumn{3}{|l|}{ Sex } & \multirow[t]{3}{*}{$p=0.730$} & \multicolumn{3}{|l|}{ Sex } & \multirow[t]{3}{*}{$p=0.459$} \\
\hline Male & 24 & 76 & & Male & 52 & 48 & \\
\hline Female & 23 & 77 & & Female & 56 & 44 & \\
\hline \multicolumn{3}{|l|}{ Age (years) } & \multirow[t]{7}{*}{$p<0.001$} & \multicolumn{3}{|l|}{ Age (years) } & \multirow[t]{7}{*}{$p<0.001$} \\
\hline $18-24$ & 0 & 100 & & $18-24$ & 17 & 83 & \\
\hline $25-40$ & 6 & 94 & & $25-40$ & 24 & 76 & \\
\hline $41-55$ & 13 & 87 & & $41-55$ & 48 & 52 & \\
\hline $56-70$ & 25 & 75 & & $56-70$ & 67 & 33 & \\
\hline $71-85$ & 60 & 40 & & $71-85$ & 74 & 26 & \\
\hline$>85$ & 100 & 0 & & $>85$ & 100 & 0 & \\
\hline \multicolumn{3}{|l|}{ Race } & \multirow[t]{6}{*}{$p=0.991$} & \multicolumn{3}{|l|}{ Race } & \multirow[t]{6}{*}{$p=0.234$} \\
\hline African American & 25 & 75 & & African American & 64 & 36 & \\
\hline Caucasian & 25 & 75 & & Caucasian & 50 & 50 & \\
\hline Asian & 17 & 83 & & Asian & 67 & 33 & \\
\hline Hispanic & 25 & 75 & & Hispanic & 50 & 50 & \\
\hline Other & 27 & 73 & & Other & 45 & 55 & \\
\hline \multicolumn{3}{|c|}{ Income (thousands of USD) } & \multirow[t]{5}{*}{$p<0.001$} & \multicolumn{3}{|c|}{ Income (thousands of USD) } & \multirow[t]{5}{*}{$p=0.01$} \\
\hline$<30$ & 28 & 72 & & $<30$ & 64 & 36 & \\
\hline 30-49 & 30 & 70 & & $30-49$ & 53 & 47 & \\
\hline $50-74$ & 7 & 93 & & $50-74$ & 51 & 49 & \\
\hline$>75$ & 6 & 97 & & $>75$ & 37 & 63 & \\
\hline \multicolumn{3}{|l|}{ Education } & \multirow[t]{6}{*}{$p=0.008$} & Education & & & \multirow[t]{6}{*}{$p<0.001$} \\
\hline $\begin{array}{l}\text { Less than high } \\
\text { school }\end{array}$ & 44 & 56 & & $\begin{array}{l}\text { Less than high } \\
\text { school }\end{array}$ & 88 & 12 & \\
\hline $\begin{array}{l}\text { High school or } \\
\text { GED }\end{array}$ & 27 & 73 & & $\begin{array}{l}\text { High school or } \\
\text { GED }\end{array}$ & 63 & 37 & \\
\hline Some college & 18 & 82 & & Some college & 47 & 53 & \\
\hline Completed college & 14 & 86 & & $\begin{array}{l}\text { Completed col- } \\
\text { lege }\end{array}$ & 47 & 54 & \\
\hline Graduate degree & 13 & 87 & & Graduate degree & 39 & 61 & \\
\hline
\end{tabular}


Table 6 Order of Patient Preferences for Orthopaedic Smartphone App Features

\begin{tabular}{|l|l|l|}
\hline Feature & Rank & Raw Score \\
\hline Reminders before next appointment or surgery & 1 & 1.057 \\
\hline Review results of tests, procedures, labs, or xrays/CT/MRI & 2 & 1.022 \\
\hline Communication with physician team (texts, emails) & 3 & 1.017 \\
\hline Discharge or after-surgery instructions & 4 & 1.007 \\
\hline Physical therapy exercise instructions/pictures/videos & 5 & 993 \\
\hline Making or changing appointments & 6 & 987 \\
\hline Medication information & 7 & 969 \\
\hline Finding a doctor: information about hospital or physicians & 8 & 938 \\
\hline Information about general orthopaedic problems & 9 & 937 \\
\hline Pictures/videos explaining surgery & 10 & 926 \\
\hline
\end{tabular}




\section{References}

1. Long C, Tsay EL, Jacobo SA, Popat R, Singh K, Chang RT. Factors Associated with Patient Press Ganey Satisfaction Scores for Ophthalmology Patients. Ophthalmology 2016; 123: 242-7.

2. Mistry JB, Chughtai M, Elmallah RK, Le S, Bonutti PM, Delanois RE, et al. What Influences How Patients Rate Their Hospital After Total Hip Arthroplasty? The Journal of arthroplasty 2016; 31: 2422-5.

3. Sullivan W, DeLucia J. $2+2=7$ ? Seven things you may not know about Press Ganey Statistics. Emergency Physicians MonthlySeptember 22, 2010.

4. Baig MM, GholamHosseini H, Connolly MJ. Mobile healthcare applications: system design review, critical issues and challenges. Australas Phys Eng Sci Med 2015; 38: 23-38.

5. Andrawis JP, Muzykewicz DA, Franko OI. Mobile Device Trends in Orthopedic Surgery: Rapid Change and Future Implications. Orthopedics 2016; 39: e51-6.

6. Al-Hadithy N, Gikas PD, Al-Nammari SS. Smartphones in orthopaedics. Int Orthop 2012; 36: 1543-7.

7. Franko OI. Smartphone Apps for Orthopaedic Surgeons. Clinc Orthop Relat Res 2011; 469: 2042-8.

8. Franko OI, Bhola S. iPad apps for orthopedic surgeons. Orthopedics 2011; 34: 978-81.

9. Jenny J-Y, Bureggah A, Diesinger Y. Measurement of the knee flexion angle with smartphone applications: Which technology is better? Knee Surg Sports Traumatol Arthrosc 2015 Feb 15.

10. Peters FM, Greeff R, Goldstein N, Frey CT. Improving acetabular cup orientation in total hip arthroplasty by using smartphone technology. The Journal of arthroplasty 2012; 27: 1324-30.

11.Izatt MT, Bateman GR, Adam CJ. Evaluation of the iPhone with an acrylic sleeve versus the Scoliometer for rib hump measurement in scoliosis. Scoliosis 2012; 7: 14.

12. Ferretti A, Valeo L, Mazza D, Muliere L, Iorio P, Giovannetti G, et al. Smartphone versus knee ligament arthrometer when size does not matter. Int Orthop 2014; 38: 2197-9.

13. Lee S, Shin JJ, Haro MS, Song SH, Nho SJ. Evaluating the quality of Internet information for femoroacetabular impingement. Arthroscopy. The journal of arthroscopic \& related surgery 2014; 30: 1372-9.

14. Ockendon M, Gilbert RE. Validation of a novel smartphone accelerometer-based knee goniometer. The journal of knee surgery 2012; 25: 341-5.

15. Qiao J, Liu Z, Xu L, Wu T, Zheng X, Zhu Z, et al. Reliability Analysis of a Smartphone-aided Measurement Method for the Cobb Angle of Scoliosis. J Spinal Disord Tech 2012; 25: E88-92.

16. Shaw M, Adam CJ, Izatt MT, Licina P, Askin GN. Use of the iPhone for Cobb angle measurement in scoliosis. Eur Spine J 2012; 21: 1062-8.

17.VonHoltz LA, Hypolite KA, Carr BG, Shofer FS, Winston FK, Hanson CW, et al. Use of Mobile Apps: A Patient-centered Approach. Acad Emerg Med 2015; 22: 765-8.

18. Azevedo R, Bernardes M, Fonseca J, Lima A. Smartphone application for rheumatoid arthritis self-management: cross-sectional study revealed the usefulness, willingness to use and patients' needs. Rheumatol Int 2015; 35: 1675-85.

19. Cho MJ, Sim JL, Hwang SY. Development of Smartphone Educational Application for Patients with Coronary Artery Disease. Healthc Inform Res 2014; 20: 117-24.

20. McCartney E, Bacci JL, Ossman KL, Richardson RM, DelPizzo D, DeJames J, et al. Mobile application features sought after by patients of a regional grocery store chain pharmacy. J Am Pharm Assoc 2016; 56: 62-6.

21.Hogan NM, Kerin MJ. Smart phone apps: smart patients, steer clear. Patient education and counseling 2012; 89: 360-1.

22. Barton AJ. The regulation of mobile health applications. BMC medicine 2012; 10: 46.

23. Gulacti U, Lok U, Hatipoglu S, Polat H. An Analysis of WhatsApp Usage for Communication Between Consulting and Emergency Physicians. Journal of medical systems 2016; 40: 130.

24. Mole DJ, Fox C, Napolitano G. Electronic patient data confidentiality practices among surgical trainees: questionnaire study. Annals of the Royal College of Surgeons of England 2006; 88: 550-3.

25.Etier BE, Jr., Orr SP, Antonetti J, Thomas SB, Theiss SM. Factors impacting Press Ganey patient satisfaction scores in orthopedic surgery spine clinic. The spine journal 2016.

26. McCormick JD, Werner BC, Shimer AL. Patient-reported outcome measures in spine surgery. The Journal of the American Academy of Orthopaedic Surgeons 2013; 21: 99-107.

27. Powell J, Inglis N, Ronnie J, Large S. The characteristics and motivations of online health information seekers: cross-sectional survey and qualitative interview study. Journal of medical Internet research 2011; 13: e20.

28. Atkinson NL, Saperstein SL, Pleis J. Using the internet for health-related activities: findings from a national probability sample. Journal of medical Internet research 2009; 11: e4.

29. Fox S, Jones S. The Social Life of Health Information. Pew Internet \& American Life Project 2009, Jun 11. 
30. Woolf AD, Pfleger B. Burden of major musculoskeletal conditions. Bulletin of the World Health Organization 2003; 81: 646-56.

31. Cotten SR, Gupta SS. Characteristics of online and offline health information seekers and factors that discriminate between them. Social science \& medicine 2004; 59: 1795-806.

32. Kantar. Apple Ends 2015 as Leading Brand in U.S. \& China. Kantar World Panel 2016.

33. Wolff JL, Berger A, Clarke D, Green JA, Stametz R, Yule C, et al. Patients, care partners, and shared access to the patient portal: online practices at an integrated health system. Journal of the American Medical Informatics Association : JAMIA 2016; 23: 1150-8. 